\title{
Analysis, Design and Implementation of a General Framework for Remote Lab
}

\author{
Yasser H. Elawady \\ College of Computers and Information Technology \\ Taif University, \\ Taif, KSA.
}

\author{
A.S. Tolba \\ Faculty of Computer Studies \\ Arab Open University, \\ $H Q$, Kuwait.
}

\begin{abstract}
Hands-on laboratory experiments are a vital part of engineering and physical sciences education, having a strong impact on students' learning outcomes. With the increased usage of electronic kits in the educational laboratories, and the need for training a huge number of students on these kits, it is imperative to enable to remote access to a physical laboratory, either as part of an on-site or distance learning course. In addition to the convenience provided to students, there are also cost and safety related benefits. Many institutions can not afford the expensive equipment provided in a physical lab. Such laboratories are very convenient and effective for learning hardware design concepts and elaborating hardware-based graduation projects. This paper addresses the hardware (HW) and software (SW) tools necessary for building a general framework for remote laboratory access. The proposed system allows performing experiments remotely across the Internet via web interface as well as locally in the classroom. In addition, it introduces enhancements for the remote lab activities leading to improving its performance and makes the data transfer more secure.
\end{abstract}

\section{Keywords-}

Remote laboratory access, Virtual laboratory, FPGA, E-learning.

\section{INTRODUCTION}

Within the disciplines of Engineering and Physical Sciences, laboratory work is considered to be at the heart of learning and can have a strong impact on students' learning outcomes. Laboratorybased sessions are widely used in order to provide physical evidence of theoretical principles and to teach practical skills. When used appropriately they can enthuse, motivate and inspire students [1].

Remote laboratories embody an attractive solution to conduct live experiments from any location, obtaining time saving and reduction of costs for measurement instruments, and technical personnel. Moreover, the achievement of remote control of an instrument has proved to be an optimal solution to share an instrument among different Universities or industrial users, without the need of transporting it or outing of personnel [2]. Most of the remote labs focus on engineering laboratories as the engineering discipline contains the biggest portion of laboratory studies. In other words, engineering is an applied science. Remote laboratories are becoming widely accepted in universities for providing distance education and for augmenting traditional laboratories. There is a lot of interest in remote laboratories from pedagogical point of view [3].With the increasing popularity and use of FPGAs and Microcontrollers $(\mu \mathrm{C})$, it becomes necessary to develop a mechanism for training a broad range of computing students on FPGA and $\mu \mathrm{C}$ technologies.

This paper introduced a general framework for a proposed remote lab access that allows performing experiments remotely across the Internet via web interface. In addition, it introduces enhancements for the remote lab activities leading to improving its performance and makes the data transfer more secure.

The rest of this paper is organized as follows: Section II introduces the related work. Section III introduces the design of the remote laboratory system architecture. Section IV presents the remote lab Case Study. Section V introduces results and discussion. The conclusion summarizes the obtained results and proposes further work for enhancing the performance of remote laboratories.

\section{RELATED WORK}

Remote lab access is a relatively new concept. With the growth of the Internet and increasing connectivity, the popularity of remote labs has surged. Some interesting efforts are described below.

RMCLab [4] is a remote laboratory which targets electrical engineering courses at the University of Patras, Greece, beginning in 2004. RMCLab incorporates an FPGA in addition to "auxiliary modules" which include differential amplifiers, PLLs, ADCs, and OpAmp circuits. The labs are broken down to employ different aspects of hardware and circuit design and analysis. The hardware is connected through PCI to a host server which integrates a signal generator, oscilloscope, and other special hardware over a custom LPT interface. Augmented client-server architecture subdivides the conventional client architecture into a client and instructor client (IC), and the server architecture into an application server (AS) and resource server (RS). RMCLab's custom design is quite specific to the available resources from the University. The individual servers coordinate access with incoming client requests with available hardware resources. While such a system can supplement large class 
sizes, one key drawback is portability to other classroom environments where these exact resources do not exist.

Hashemian and Riddley [5] have designed an FPGA e-Lab, remote access system targeted for digital design courses using FPGAs based on Xilinx's Spartan-3E Starter Kit. The e-Lab uses Windows XP Remote Desktop to connect the remote user with the FPGA, data acquisition hardware, and LabView, Integrated webcam and GPIO to connect LEDs, switches, and control hardware on the actual FPGA. An obvious advantage of such a system is the low start-up costs associated with acquiring the Spartan Starter Kit and software tools, but the use of Windows XP Remote Desktop slows the system.

Nedic [6] reported on NetLab from the University of South Australia which aims to address the common concerns of remote laboratories. With a similar design to the previous efforts, NetLab incorporates a variety of laboratory equipment and even goes so far as to allow collaboration between students. The paper concludes that while the remote lab setup did not directly outperform conventional real labs, the authors suggest a mix of real and remote labs throughout the education curriculum.

Corter et al [7] developed a model that investigates the relative effectiveness of hands-on labs, remote labs and simulated labs. The labs conducted were focused on the kinematics and dynamics of mechanisms such as linkages, cams and gears. To draw a fair comparison, out of the six labs given to the class, three were given in traditional format and three were given in hands-on traditional format. In addition to the student outcomes and student satisfaction, student preferences for remote labs were related to student characteristics, in thinking style and ability. The results obtained by Corter et al show that among other criteria like preparatory instructions, lab report, and team work, "physical presence in the lab" was rated least important by the students. Also, actual learning outcomes were assessed by questions on the midterm and final that was related to the content of the labs.

Lab on the Web [8] is a virtual laboratory that aims to achieve flexibility in digital and analog circuit design keeping in mind the cost, scalability and modularity. An

FPGA board is used as it allows for implementation of almost any circuit. Here, the interface to the remote lab is achieved by combining Virtual Network Computing (VNC) and secure remote desktop. Test suites were developed that are lab experiments which can be used by students. The test suites include an Analog Circuit - a simple circuit is set up which can demonstrate the use of a power supply, oscilloscope, a switch matrix and a function generator all of which can be controlled Remotely, FPGA Programming - illustrates that the FPGA can be used Remotely via the Internet, Digital Signal Processing Experiment - An analog signal is captured then using the ADC interface it is digitized. The signal is then passed to the FPGA using VHDL. This system also provides a webcam so the users can look at the FPGA board. This system was evaluated and it was found suitable for undergraduate courses.
"eLab" [9], implemented by the University of Bordeaux in France is an effort toward increasing Remote education for Electrical Engineering. The components that make up this system are a pool of instruments that can be controlled remotely, a group of servers and dedicated software. The digital circuits implemented by this lab include Differential Pair Amplifier, Linear OpAmp operation, and RC filter. Again, a camera is placed in the eLab's room that allows a live view of the instruments and servers. The eLab webpage also contains various textbooks that are required for the courses that use these labs. The web interface is a simple one that allows users to set data points, frequency values and voltages. The results of these inputs can be then measured in new pop-up window which allows users to save the output. An interesting feature in this system is that a "notebook" is automatically created that stores the results and measurements of the user.

Gurkan et. al [10] presented a remote laboratory for an optical circuit's course. The strategy used in this facility is to first introduce the students to the concepts in theory and then proceed to pre laboratory activities. The pre laboratories include an orientation video, simulation and laboratory procedures on-line. This work also includes assessment of the learning outcomes and teaching methods. Here, the students connect to the server using a Web-based client that connects to the LabView Web Server. Three experiments were conducted; Optical Source Characterization, Optical Fiber Link Attenuation and Fiber Connectors, Hands-On Skills Transferred From RemoteLabs, based on performance of the students on these experiments, student success was measured. A student opinion survey was conducted too.

Austin J. Che [11] at the Massachusetts Institute of Technology (MIT), explains the benefits of setting up remote lab for biology using the iLab technology developed by MIT. Che states that a general lab for biology that can be done remotely, leads to shared costs, efficient use of resources and higher work efficiency.

From the above descriptions, it can be noted that many researchers are investigating the effectiveness of remote labs in their respective fields. Many of the labs implement FPGAs due to their flexibility and reconfigurability, but the number of remote labs for FPGA education is relatively very few. The target of this paper is to widen FPGA education and other electronic courses based on laboratory kits. Also, the use of a webcam in this project is necessary to make reality to the presence in the lab. Another interesting fact to note is the evaluation methods used in these facilities. Since there is no standard method of proving the effectiveness, it can be seen that the trend is to collect feedback from the students using the facility. Although, anecdotal data is arguably not the strongest means of proving facts, it is very helpful to improve the facility. Another method is to test the students and drive learning outcomes. From the standardization point of view, the most significant difference of the work described in this paper is that it is comparatively more scalable in the sense that the hardware and software are both flexible to fit in different environments. 


\section{DESIGN OF REMOTE LABORATORY SYSTEM ARCHITECTURE}

\subsection{System Architecture}

The structure of the proposed remote lab access is shown in Fig.1. The system simply represents the remote lab with single structure of a computer to interact with the client (student) through the internet via a VPN connection. It consists of two sections, the user domain and the remote lab. The user domain enables the client in his place outside the lab to send his programs and data to the remote lab to be executed and installed on the kits connected to the remote lab manager. The second section, the remote lab, is simply the physical location for the client to execute his experiments on the kits connected to the remote lab manager through a permission access to the lab remotely.

There are data encrypting and decrypting layers in both sections to improve the security of data transfer.

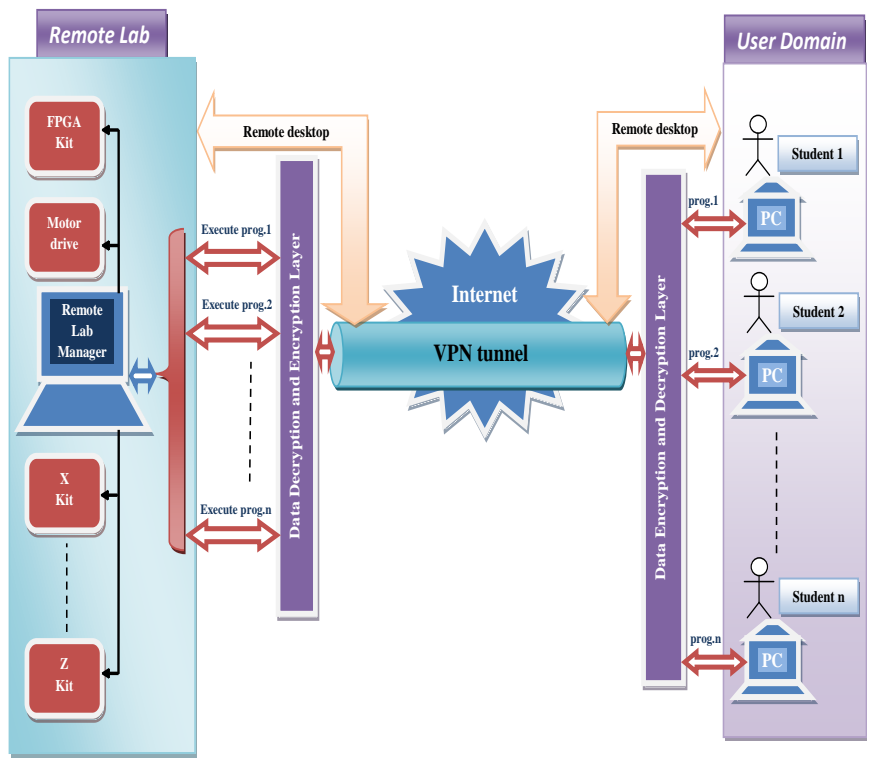

Fig.1.Remote Lab Structure

\subsection{Remote lab Hardware}

From the standardization point of view, the hardware requirements of the remote lab in terms of the experimentation unit are dependent on the type of the experiment to be conducted. The remote lab manager, power supply unit, the electronic kits, some aided electronic circuits to connect the kits to remote lab manager

terminal (i.e. USB terminal), and internet connection are typical laboratory instruments needed. They may be augmented or changed based on the type of the experiment needed to be set-up. The main requirement is that the measuring device be accessible via some wellknown interface for control selection and setup, as well as remote data collection.

In our remote lab we have three types of kits as follow:
i. FPGA kits (Xilinx Spartan_3A)
ii. Microcontroller PIC_16F876 for Motor drive.
iii. Wireless sensors (temperature, light)

They are connected to the remote lab manager through USB cables. The remote lab can be extended to have more kits simply by connecting these kits to the remote lab manager and installing the driver of these kits on the remote lab manager.

\subsection{Remote lab Software}

Our remote lab is programmed with $\mathrm{C \#}$ and the software needed to run the kits are different due to the kit chosen to be run by the client. Each kit has its own driver and installed on the remote lab manager. The client connects to the lab through internet in a secured tunnel with VPN technology. Our remote lab is a client/server package that contains two applications:

- Lab Manager Application.

- Student Front-end application.

\subsubsection{Lab Manager Application:}

This application is a windows application that works as a server; the lab instructor is the administrator of this application. The operations will be done locally on the lab manager machine.

The application has the following main features:

1- Connection Administration.

2- Student administration.

3- Work Space administration.

4- Lab Terminals Administration.

5- Executing Experiments.

6- Network-Fail Safety protocol.

\subsubsection{Student Front-end Application:}

This application works as a client and student is the user of this application. The student can do these operations:

1. Login to the Lab Manager.

2. Upload/Edit/Delete Experiments in his work space.

3. Execute experiments.

4. Get Remote desktop. 


\subsection{Object-Oriented Design of the proposed system .}

A software system can be said to have two distinct characteristics: a structural, "static" part and a behavioral, "dynamic" part. In addition to these two characteristics, an additional characteristic that a software system possesses is related to implementation. The static characteristic of a system is essentially the structural aspect of the system that defines what parts the system is made up of. It includes Use case diagram and Class diagram. The dynamic characteristics of a system are essentially the behavioral features of that system including Object diagram, State diagram, Activity diagram, Sequence diagram, and Collaboration diagram.

The implementation characteristic of a system describes the different elements required for deploying a system, including Component diagram and Deployment diagram [12].

For the proposed system, we presented the two structural components (Use case diagram and Class diagram), and two behavioral features (Sequence diagram and Activity diagram), using Microsoft Visio 2010 for depicting these diagrams.

\subsubsection{Remote Lab Use Cases}

The use cases of the remote lab are shown in Fig.2.

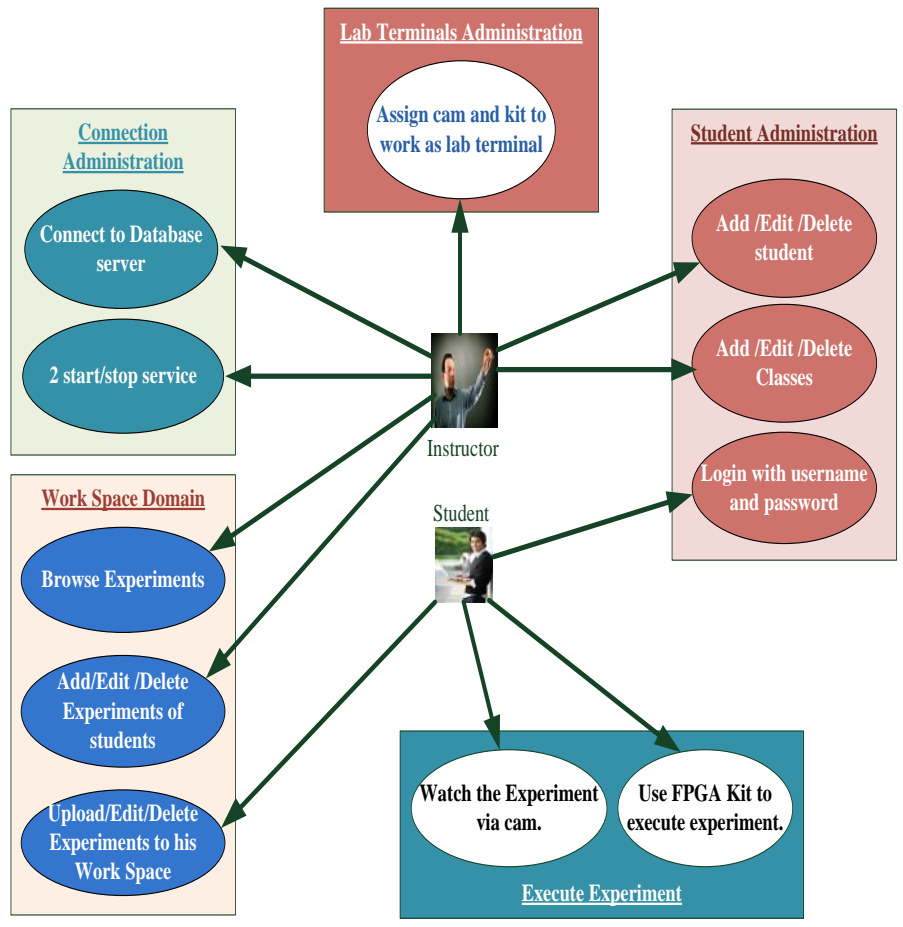

Fig.2.Remote Lab Use Cases

\subsubsection{Remote Lab Activity diagram}

The remote lab activity diagram is shown in Fig.3

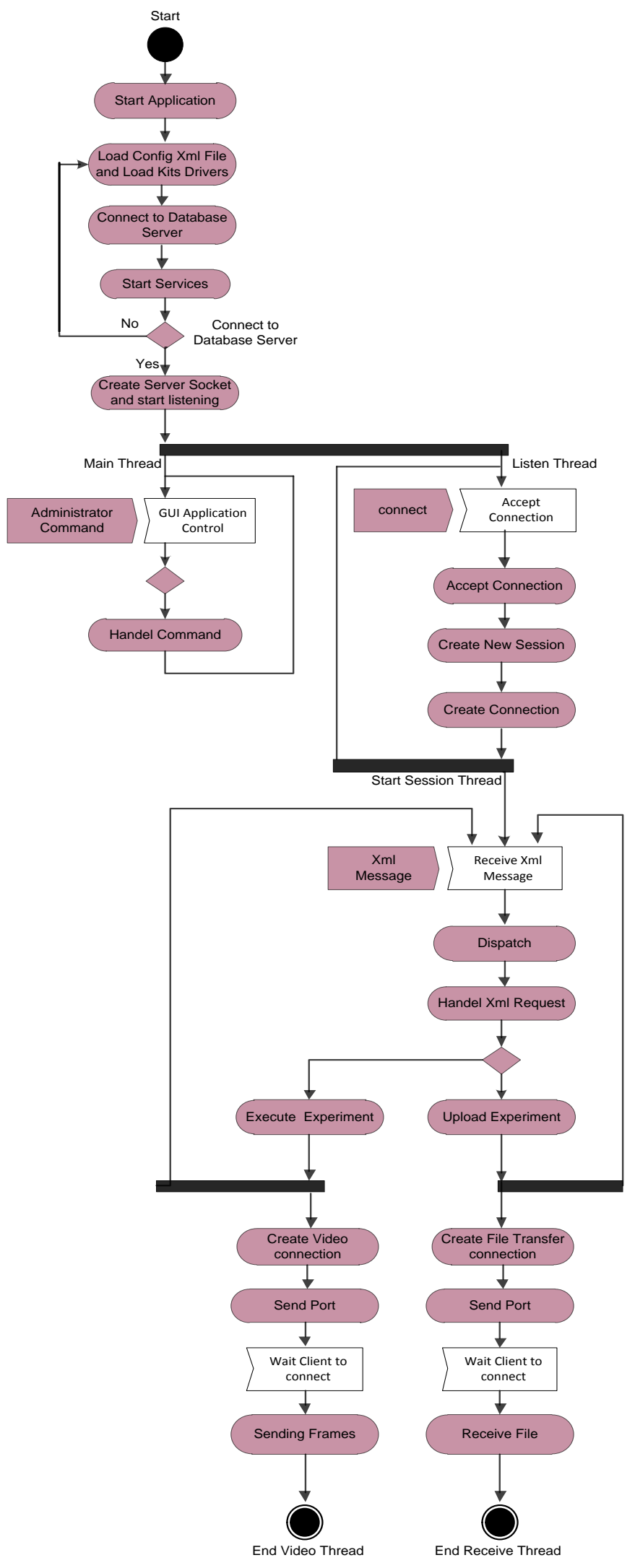

Fig.3 The Remote Lab activity diagram. 


\subsubsection{Remote -Lab Class Diagram}

The overall class diagram of the remote lab access is shown in Fig.4

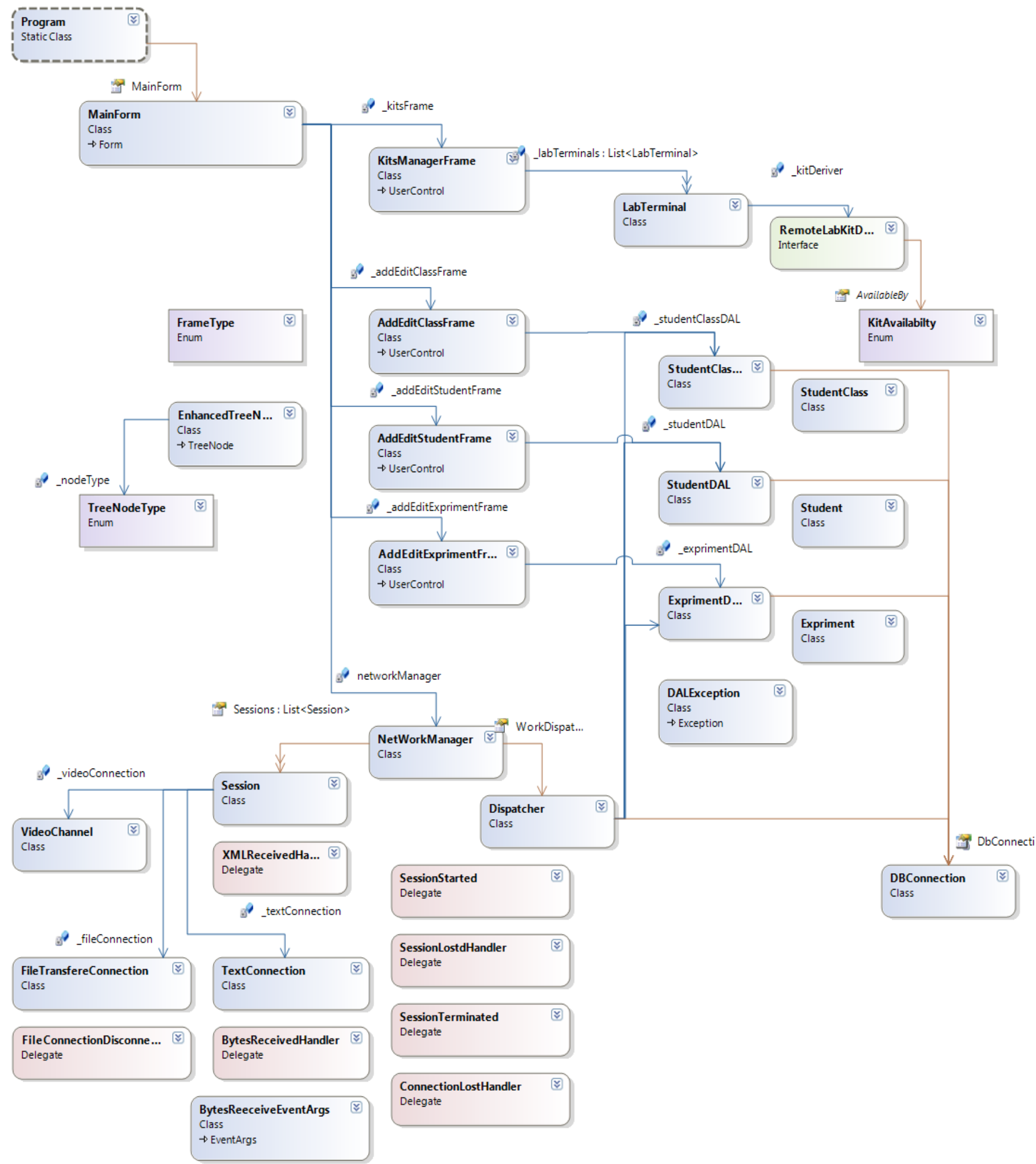

Fig.4 Overall Class Diagram Of The Remote Lab. 


\subsubsection{Remote Lab Sequence Diagram}

The remote lab sequence diagram is shown in Fig.5

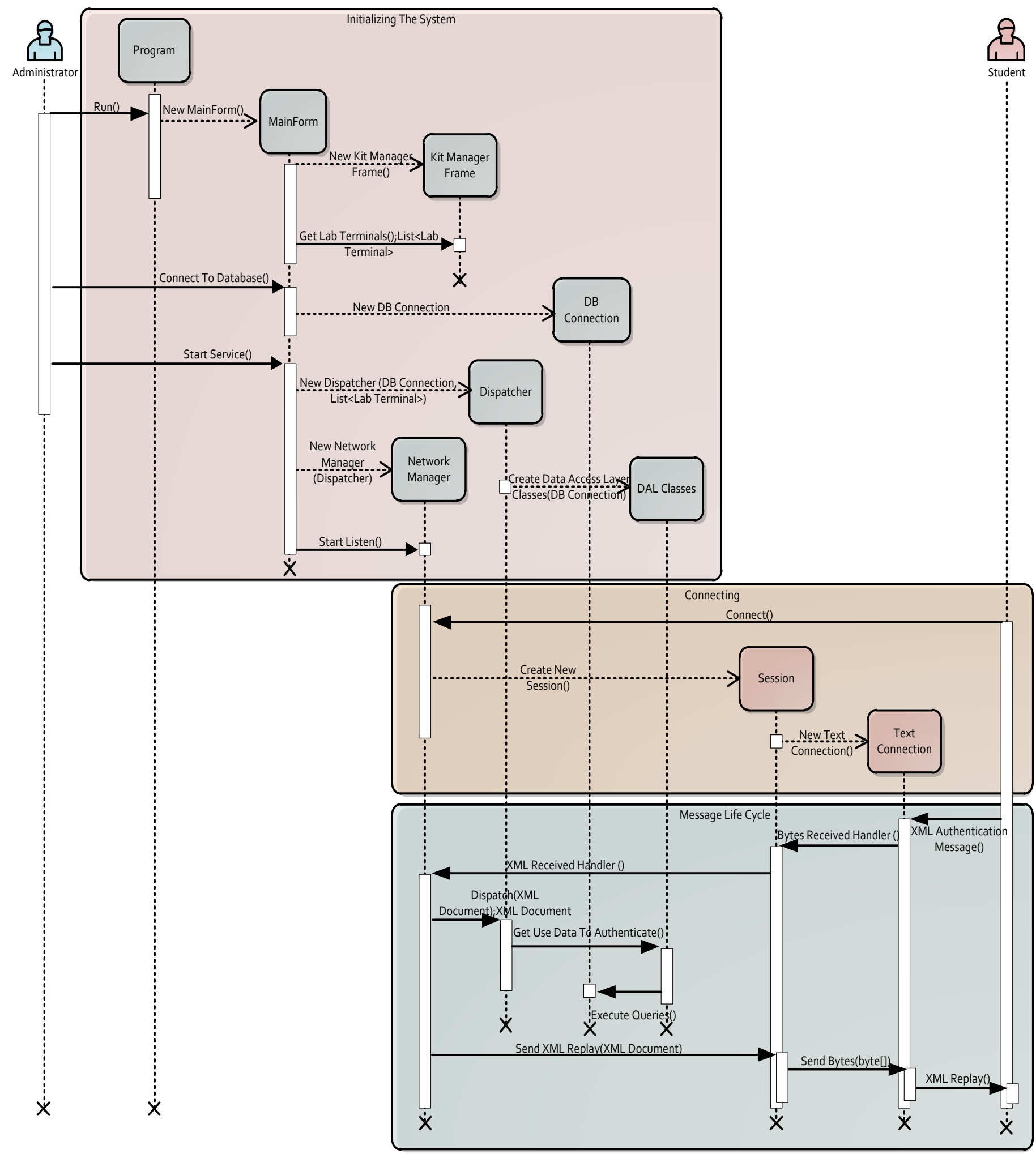

Fig.5 Remote Lab Sequence Diagram 


\subsection{Data Encryption}

For securing data transfer an Encryption-Decryption layer is built at both sides of the proposed remote lab.

The policy of the Data Encryption-Decryption in the proposed remote lab is as follow:

i. Server application has RSA keys (public and private key).

ii. Client application encrypt all vital small data with server public key so only the server application can decrypt and get the data.

iii. When client application needs to send large amount of data (like files) it generates symmetric key like Text To Image (TTI) and encrypt this key by public key and send it to the server then server can decrypt it by private key and get the symmetric key. Client application will encrypt this file with symmetric key and send it to the server.

The Data Encryption-Decryption Sequence Diagram is shown in Fig. 6

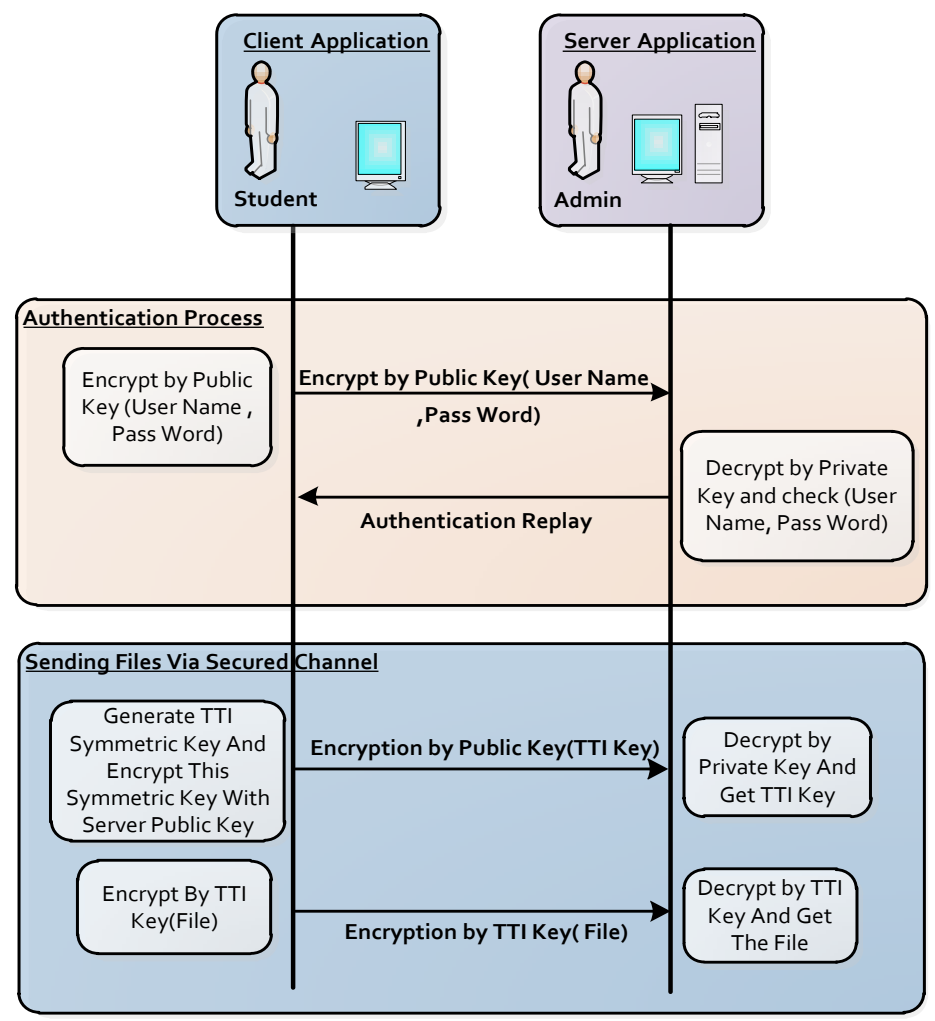

Fig.6 Data Encryption Sequence Diagram

\section{REMOTE LAB CASE STUDY}

The remote lab has two modes of operation available for the choice of the client as follow:

i. Direct execution
(The client writs the program on his home PC then he uploads the file to the remote lab manager remotely and download this (.bin) file to the kit through the port connection with remote lab manager, Remote desktop not needed)

\section{ii. Remote desktop}

(The client starts programming the file on the remote Lab manager remotely then downloads this file to the kit)

In the next section we will introduce a case study for direct execution mode of the proposed remote lab with FPGA design and test using Xilinx Spartan_3A kit.

\subsection{FPGA Design And Test Using Xilinx Spartan_3A Kit}

Modern large FPGA devices have capacity equivalent to millions of equivalent gates and contain big amount of embedded multipliers, Digital Signal Processing (DSP) blocks, hierarchical memory subsystems, hard cores etc. The proposed remote lab is equipped with a Xilinx Spartan-3A Starter Kit whose hardware is interfaced with a remote lab manager via an interactive Graphical User Interface (GUI) and acquisition hardware through USB ports. The access to the remote lab manager from a Remote location will be through a VPN connection for more secure data transfer.

A typical FPGA design procedure includes the following steps:

1. Project description and specification.

2. Design entry through schematic capture and/or Hardware Description Languages such as VHDL or Verilog.

3. Functional simulation and design verification.

4. Design synthesis.

5. Design implementation, post place and route simulation.

6. FPGA hardware reconfiguration.

7. Design verification: testing and debugging.

Stages 1 through 5 can be accomplished using only Electronic Design Automation (EDA) software tools. Stages 6 and 7 require access to the real FPGA hardware.

In direct execution mode the client will carry out the first 5 stages to get the program and then completes the process by executing Stages 6 and 7 on the real FPGA hardware in the remote lab. In this mode, the client uploads the (.bit) file to the work space, and downloads it to the FPGA kit using a batch file for the impact program such as the following example.

Example: Full-adder

1- Run client application.

2- Login to the lab using (class number, user name and password) as shown in Fig.7. 
3- Add experiment by inserting (experiment name, experiment description and upload (.bit) file to the work space) as shown in Fig.8.

4- The client executes the experiment on the server.

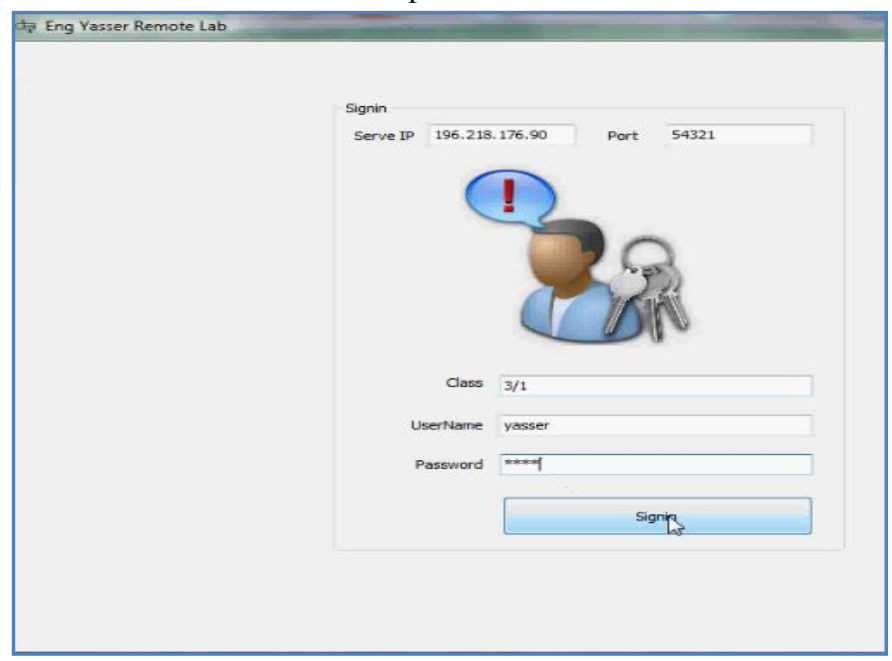

Fig.7. Login screen

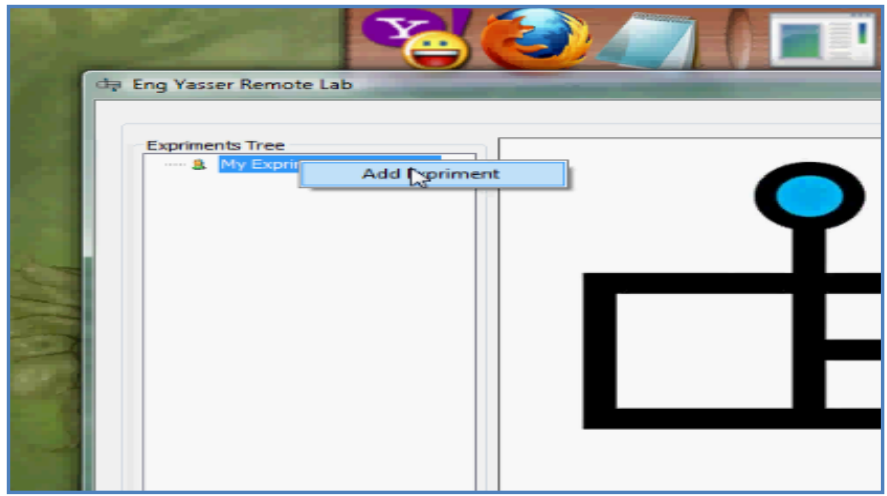

Fig.8 Add Experiment Screen.

5- The client Selects the kit and chooses execute as shown in Fig.9

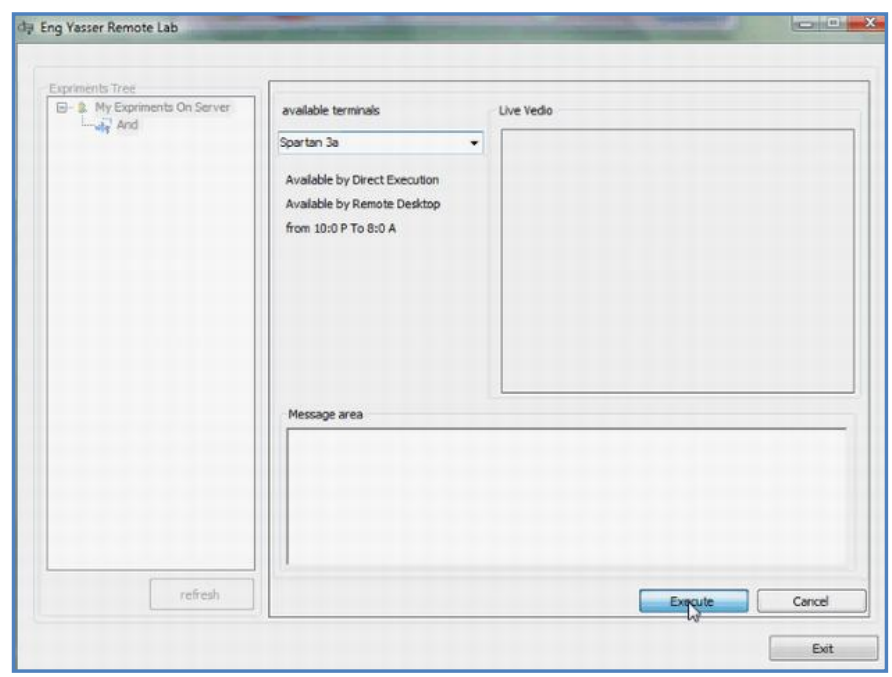

Fig.9 Select Kit and Execute Screen.
6- The client will receive success message in the message area after program download on the kit is completed. From live video, the kit led will light to ensure that the process is completed as shown in Fig. 10

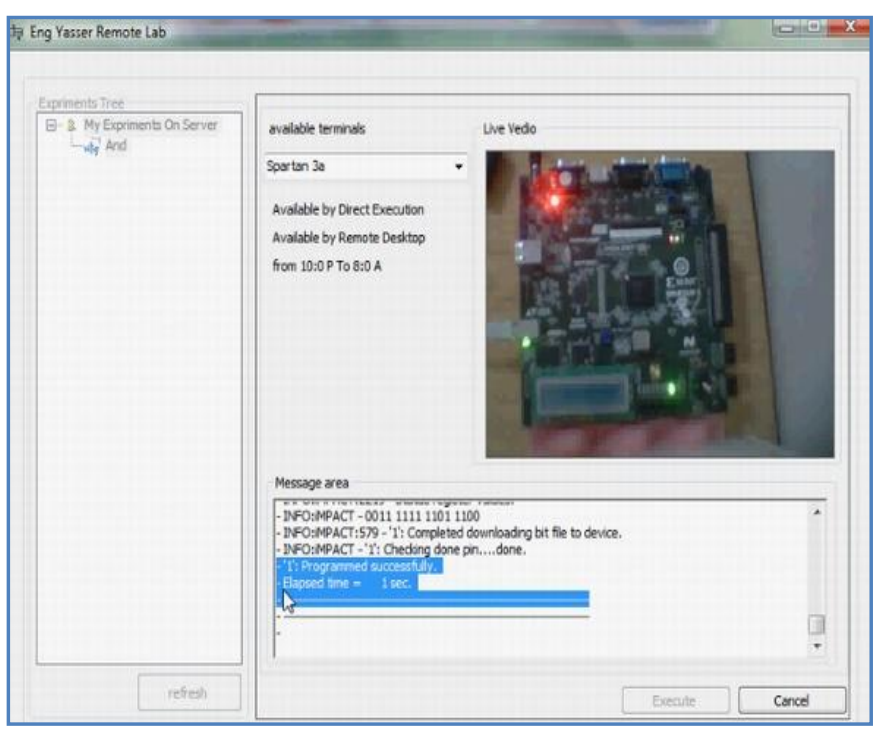

Fig.10 Success Message and Live Video Screen.

\section{RESULTS AND DISCUSSION}

As a case study of up to five students have an access to the remote lab manager at the same time, we test the average waiting time for the students to execute their experiments in the two modes remote desktop (ordinary mode in most of remote labs) and direct mode of the proposed remote lab.

The bar chart in Fig.11 shows that the direct execution reduces waiting time for the clients and enhances lab performance.

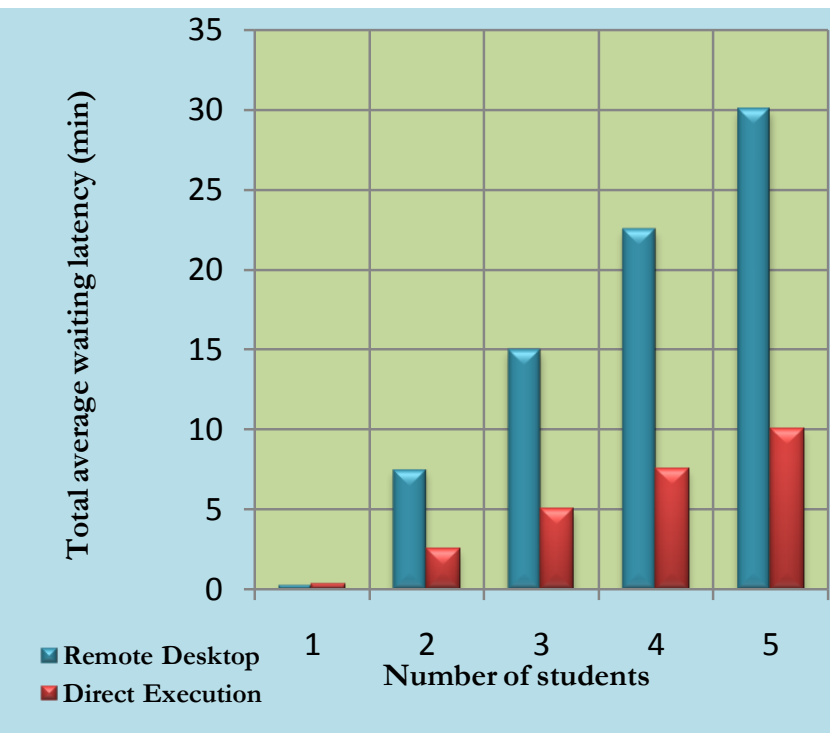

Fig .11 Number of students versus average waiting time. 
A comparative analysis of the two modes of the remote lab access is shown in table 1.

Table 1 A comparison between the two modes of the remote lab

\begin{tabular}{|l|l|l|}
\hline & \multicolumn{1}{|c|}{ Remote Desktop } & \multicolumn{1}{c|}{ Direct Execution } \\
\hline Speed & Slow & Very Fast \\
\hline $\begin{array}{l}\text { Machine } \\
\text { Resources }\end{array}$ & $\begin{array}{l}\text { Needs high machine } \\
\text { resources and high } \\
\text { speed internet } \\
\text { connection }\end{array}$ & $\begin{array}{l}\text { Doesn't Need high } \\
\text { machine resource } \\
\text { and low speed } \\
\text { internet connection } \\
\text { can Work }\end{array}$ \\
\hline $\begin{array}{l}\text { Users Can } \\
\text { Lab }\end{array}$ & Only One user & More than one user \\
\hline Security & low & high \\
\hline
\end{tabular}

\section{CONCLUSION}

In conclusion, we proposed a general framework for remote laboratory which enhances the remote lab activities leading to improving its performance and makes the data transfer more secure. So, the following conclusions are drawn,

- Remote lab access reduces the waiting time of accessing the lab through direct execution users.

- $\quad$ Remote lab access is more secure for data transfer using VPN technology (tunnel), RSA for (User Name and Pass Word), and TTI encryption for the file uploaded to the lab.

From the standardization point of view, the most significant difference of the work described in this paper is that it is comparatively more scalable in the sense that the hardware and software are both flexible to fit in different environments.

As a future work, for further analysis, the concept of Internet Of Things (IOT) can be employed to increase the interactivity between the components of the remote lab.

\section{REFERENCES}

[1] Ben Hanson, Peter Culmer, Justin Gallagher, Kate Page, Elizabeth Read, Andrew Weightman and Martin Levesley, "ReLOAD: Real Laboratories Operated At Distance", IEEE Transactions on Learning Technologies, November, 2009.

[2] Landi, Liccardo and Polese, "Remote Laboratory Activities to Support Experimental Session for Undergraduate Measurements Courses", IMTC - Instrumentation and Measurement, Technology Conference, Sorrento, Italy, April, 2006.

[3] Dervis Deniz, Atilla Bulancak and Gökhan Özcan, "A Novel Approach to Remote Laboratories", 33rd ASEE/IEEE Frontiers in Education Conference, Nov., 2003.
[4] Karadimas and Efstathiou, "An integrated platform, implementing real, remote lab-experiments for electrical engineering courses". In WBED'07: Proceedings of the sixth conference on IASTED International Conference Web-Based Education, Anaheim, CA, USA, 2007. ACTA Press.

[5] Hashemian and Riddley, "FPGA e-lab, a technique to remote access a laboratory to design and test". IEEE International Conference on Microelectronic Systems Education, MSE07, June 2007.

[6] Nedic, Machotka, and Nafalski, "Remote laboratories versus virtual and real laboratories". Frontiers in Education (FIE 2003) 33rd Annual, 2003.

[7] Corter, Nickerson, Esche, Chassapis, S. Im, and J. Ma.,"Constructing reality: A study of remote, hands-on, and simulated laboratories". ACM Transaction, Computer-Human Interaction, 2007.

[8] Pawan, Pepic, Wong, and Gulak, "Lab on the web". In Proceedings of the 4th IEEE International Conference for Upcoming Engineers (ICUE), volume 4. IEEE, May 2005.

[9] Zimmer, Billaud, and Geoffroy, "A remote laboratory for electrical engineering education". In Proceedings of the 23rd International Conference on Machine Computing, Washington DC, USA, 2006, IEEE Computer Society.

[10] Gurkan, Mickelson, and Benhaddou,"Remote laboratories for optical Circuits", IEEE Transactions on Education, Feb. 2008.

[11] A. Che., "Remote biology labs". In Education without borders, IEEE Computer Society, Washington DC, USA, 2005.

[12] Mandar Chitnis, Pravin Tiwari, and Lakshmi Ananthamurthy, "UML Overview", 2010. www.bscheele.com/xhtml/classes/ssd3/ssd3/uml_handout.doc

[13] Zafer Aydogmus, and Omur Aydogmus, "A Web-Based Remote Access Laboratory Using SCADA", IEEE Transactions on Education, Vol. 52, No. 1, February 2009

[14] Mario Bochicchio and Antonella Longo "Hands on Remote Labs: Collaborative Web Laboratories as a Case Study for IT Engineering Classes", Accepted by IEEE Transactions On Learning Technologies, 2010

[15] Milos Drutarovsky, Jan Saliga and Ingrid Hroncova," Hardware Infrastructure Of Remote Laboratory For Experimental Testing Of FPGA Based Complex Reconfigurable Systems", Acta_Electrotechnique and Information Vol. 9, No. $1,2009$.

\section{AUTHORS PROFILE}

Yasser H. Elawady is a Lecturer in the Department of Computer Engineering, Faculty of Computers and Information technology, Taif University, Taif, KSA. He received his M.Sc. from the Department of Computer Engineering, Faculty of Engineering, Mansoura University, Mansoura, Egypt, in 2003. His subject of interest includes Remote Access, FPGA programming, Hardware Design, computer architecture and organization and Networking.

A. S. Tolba is a full professor at Mansoura University, Egypt. He is a professor of Computer Science and Engineering. Professor Tolba holds a $\mathrm{PhD}$ in Computer Vision from Wuppertal University in Germany. He is the author of over 70 papers that have been published in refereed international Journals (Computers in Industry, 
Cybernetics and Systems, Digital Signal Processing, Pattern Analysis and Applications, Biomedical Research, International Journal of Hybrid Intelligent Systems, International Journal of signal Processing, International Journal of Computers and Applications and IJCSNS International Journal of Computer Science and Network Security) and conferences in the areas of Face Recognition, Neural Networks, Glove-Based Gesture Recognition, Speaker Recognition, MRI Compression, Data mining, Automated Visual Inspection of Flat Surfaces, Combined Classifiers, Signature Recognition and eLearning. He has served as the director of the national project "ICT in Higher Education Development in the Egyptian Universities". He is the founder and director of the eLearning Center at Mansoura University, Egypt during the period (2005-2007) and served as the Director of the National eLearning Center (20062007).
Professional activities include Deputy Dean of Graduate Studies and Research (2003), Dean of the Faculty of Computer Science and Information Systems in Mansoura University (2004-2007) and he is currently, the Dean of the Faculty of Computer Studies at the Arab Open University, Kuwait. He served as an IT and Educational Technology consultant for the minister of general education in Egypt in 2006. He served also as a reviewer in many international Journals: IEEE Transaction on Pattern Analysis and Machine Intelligence, Image Processing and Vision Computing. He has published books in Computer Vision and Robotics, Edited Book, 1990 (Kluwer Academic Publishers), Lasers in Computing and Health (ALESCO, Morocco, 1997), ICTs and Higher Education in Africa,2007, Published under a Creative Commons License, 2007, and E-learning in General Education, 2008 (The Arab network for Open and Distance Education, National Library, Jordan). 